Bien-être animal et protection des minorités religieuses en Belgique. Le test de l'abattage rituel entre écarts régionaux et attente constitutionnelle

\title{
Louis-Léon Christians
}

\section{(2) OpenEdition}

\section{Journals}

Édition électronique

URL : https://journals.openedition.org/rdr/1704

DOI : $10.4000 /$ rdr. 1704

ISSN : 2534-7462

Éditeur

Presses universitaires de Strasbourg

\section{Édition imprimée}

Date de publication : 2 décembre 2021

Pagination : 79-99

ISBN : 979-10-344-0097-3

ISSN : 2493-8637

\section{Référence électronique}

Louis-Léon Christians, «Bien-être animal et protection des minorités religieuses en Belgique. Le test de l'abattage rituel entre écarts régionaux et attente constitutionnelle», Revue du droit des religions [En ligne], 12 | 2021, mis en ligne le 02 décembre 2021, consulté le 03 mai 2022. URL : http:// journals.openedition.org/rdr/1704; DOI : https://doi.org/10.4000/rdr.1704

La revue du droit des religions est mise à disposition selon les termes de la Creative Commons Attribution - Pas d'Utilisation Commerciale 4.0 International - CC BY-NC 4.0. 


\section{Bien-être animal et protection des minorités religieuses en Belgique. Le test de l'abattage rituel entre écarts régionaux et attente constitutionnelle*}

\section{Louis-Léon CHRISTIANS}

Chaire Droit \& Religions, Université catholique de Louvain

\section{RÉSUMÉ}

Les évolutions régionalistes du droit belge permettent d'observer que le bien-être animal, et en particulier la question de l'abattage rituel, font l'objet en 2021 de réponses différentiées, voire discordantes, à l'interne même des structures institutionnelles belges. Il n'y va pas seulement d'une tension entre bien-être animal, protection des minorités religieuses et privilèges culturels dominants, il y va aussi de débats parlementaires qui reconduisent de nouvelles distinctions entre religions et s'en remettent de façon peu interrogée aux grilles de la globalisation de l'économie alimentaire.

\section{Abstract}

The regional developments in Belgian law show that animal welfare, and in particular the issue of ritual slaughter, are the subject of differentiated, even discordant, responses within Belgian institutional structures in 2021. Not only is there a tension between animal welfare, the protection of religious minorities and dominant cultural privileges, but also parliamentary debates reiterate new distinctions between religions and rely in an unquestioning way on the grids of the globalization of the food economy.

* Ce texte constitue la version abrégée d'une contribution en cours de publication à la Revue belge de droit constitutionnel, 2021. 
$\mathrm{U}$ n ouvrage juridique belge récent consacré aux droits des animaux s'ouvre d'emblée, dans sa préface ${ }^{1}$, par une référence à la Bible et aux grandes traditions religieuses, fondatrices des liens premiers entre l'humain et l'animal. Sans doute y rappelle-t-on que ces liens ne sont pas univoques. Entre le respect de la création et la domination de celle-ci, la ligne d'équilibre a toujours été complexe. Cet ouvrage dense ne consacre cependant par la suite aucune place significative à des contentieux liés à la religion, ni à la question, par exemple, de l'abattage rituel et à ses relations avec le bienêtre animal. Ce n'est nullement dire que cette question soit ignorée de la société belge, mais peut-être seulement que la question religieuse demeure un épiphénomène dans le dossier bien réel du statut de l'animal et de son bien-être. Un épiphénomène quantitatif, ne concernant qu'une très faible part de la consommation nationale de viande (liée aux besoins d'une partie religieuse juive ou musulmane représentant six pour cent de la population belge $^{2}$ ), mais une question devenue autant symbolique que polémique, tant dans le chef des associations de défense animale qu'auprès de certains partis politiques $^{3}$. Le contentieux judiciaire s'est renforcé lentement depuis les années 2000, et, à la suite de restrictions législatives partielles, en 2017, s'est déployé en contentieux constitutionnel et européen.

Le Conseil d'État de Belgique, en sa section législation, a réaffirmé à de nombreuses reprises que l'équilibre entre la priorité à reconnaître aux droits humains de liberté de religion et l'amélioration progressive des intérêts des animaux ne pouvait se jouer de façon binaire, mais appelait des processus

1. P.H. Delvaux, «Préface», in F. Dossche (éd.), Le droit des animaux. Perspectives d'avenir, Bruxelles, Larcier, 2019, p. 11-15. On renverra également en particulier à C. SAGESSER, «Les débats autour de l'interdiction de l'abattage rituel », Courrier hebdomadaire du CRISP, $n^{\circ}$ 2018/20. Pour l'analyse du droit régional flamand, à Ch. DE COSTER, «Hoe kossjer is onverdoofd slachten», Rechtskundig Weekblad, no 40, 2016, p. 1563-1581; E. VleUGELS et E. TAVERnA, «Het Vlaams verbod op onverdoofd slachten: geen dode letter», Tijdschrift voor Bestuurswetenschappen en Publiek Recht (TBP), $\mathrm{n}^{\circ}$ 6, 2021, p. 316-332.

2. «En Wallonie, en 2014, l'abattage selon les rites religieux concernait quelque $6,35 \%$ des 190495 abattages de bovins et 35,8\% des 13282 abattages d'ovins $»$ : CONSEIL WALLON DU BIEN-ÊTRE DES ANIMAUX, Avis relatif aux abattages sans étourdissement, 31 mai 2016: http:// bienetreanimal.wallonie.be/files/documents/CWBEA-abattage-etourdissement.pdf [consulté le 21 juin 2021]. La même année, le pourcentage d'animaux abattus sans étourdissement en Flandre s'établissait à 13,9\% des boufs, $10,5 \%$ des veaux et $96,1 \%$ des moutons: P. VANTHEMSCHE, Rapport over de dialoog met de geloofsgemeenschappen met het oog op een significante verbetering van het dierenwelzijn bij de praktijk van ritueel slachten en de overgang naar een algemeen verbod op onbedwelmd slachten, 2017, p. 31.

3. V. F. Dassetto, M.-N. Hennart, « Rite sacrificiel dans la ville de consommation. Pratiques et signification de l'Aïd El Kebir: quelques aspects », in F. DASSETto (éd.), Facettes de l'Islam belge, Bruxelles, Bruylant, 1997, p. 200-211. 
d'auto-transformation et d'évolution, en coopération avec les acteurs collectifs impliqués ${ }^{4}$.

Telle n'a pas été la réponse politique au contraire très polarisée qui s'est manifestée dans deux des trois Régions de Belgique: la Flandre et la Wallonie. La cause animale et la question de l'intégration islamique s'y sont exacerbées, tandis que la population juive, préservée depuis la seconde guerre mondiale, s'est considérée comme victime collatérale d'un contentieux s'adressant principalement à l'islam, et occasion pour certains d'une nouvelle forme d'antisémitisme. Seule la Région de Bruxelles-Capitale, dont la population concentre une part importante des habitants musulmans de Belgique, s'est à ce jour (janvier 2021) abstenue de contrarier les usages religieux d'un abattage sans étourdissement ${ }^{5}$.

Quels sont les éléments spécifiques qui caractérisent les débats juridiques belges ${ }^{6}$ ? Quels enseignements originaux pourraient-ils apporter à une question aussi transversale en Europe du Nord-Ouest?

Après une évolution très lente de 1929 à 2015, durant lequel l'intérêt du législateur pour le bien-être animal va se déployer très progressivement, mais avec une constante volonté de préserver les usages religieux minoritaires juifs, une seconde période, très rapide, de 2015 à nos jours, s'est caractérisée par une rupture de rythme et une focalisation sur la question religieuse de l'abattage rituel sans étourdissement. À défaut, pendant longtemps, de toute jurisprudence significative, il a paru intéressant de porter une attention plus précise aux arguments échangés dans le processus d'élaboration des normes, entre débats parlementaires et avis de la section législation du Conseil d'État. On percevra à quel point le contraste s'est creusé entre les légistes et les juristes. Par-delà la complexité des arguments et des enjeux, la question du bien-être animal s'est faite révélatrice des rapports toujours renouvelés entre majorité et minorité, humaine ou animale. On observera notamment une querelle de méthode entre deux conceptions de la loi: la loi comme conciliation et la loi comme injonction, querelle particulièrement

4. Le Conseil d'État, section de législation, a rendu six avis dans ce même sens: $\mathrm{n}^{\circ} 40.350$ (2006, en Assemblée générale), 57.522 (2015), 56.484 (2016), 59.485 (2016), 69.870 (2017), 69.871 (2017): http://www.raadvst-consetat.be [consulté le 21 juin 2021].

5. Sans que la Région de Bruxelles-Capitale ne se désintéresse nullement de la question du bien-être animal: V. A. GUISSART, «La répression de la violence envers les animaux dans la Région bruxelloise», Journal des tribunaux 2020, p. 695-701.

6. Pour un examen du contexte juridique belge récent, V. L.-L. Christians et L. Vanbellingen, «Les évolutions récentes du droit belge dans la régulation du fait religieux (2015-2019)», Revue du droit des religions, $\mathrm{n}^{\circ} 8,2019$, p. 191-207. 
vive à un moment où la réaffirmation de la primauté de la loi civile semble devenir anxiogène au sein du débat public, et conduit à limiter la légitimité du principe de proportionnalité. On soulignera enfin, en conclusion, combien les niveaux de territorialité du droit viennent rouvrir le débat: un premier aspect tenant à la structure régionalisée du droit belge, un second tenant au principe européen de libre circulation, un troisième tenant au phénomène contemporain de globalisation du droit (et des religions...).

\section{BIEN-ÊTRE ANIMAL EN BELGIQUE : LE TOURNANT RÉGIONALISTE}

Deux événements juridiques quasi concomitants vont être l'occasion de relancer les mutations du droit belge en matière de bien-être animal ${ }^{7}$.

Il s'agit d'une part de l'évolution du droit de l'Union européenne concernant le bien-être animal: notamment le règlement (CE) $\mathrm{n}^{\circ}$ 1099/2009 du Conseil du 24 septembre $2009^{8}$, entré en vigueur en 2013, qui impose une série de nouvelles contraintes aux États, mais continue à rendre possible une exception au bénéfice de l'abattage rituel (question européenne qui est traitée ailleurs dans ce numéro). Et d'autre part, la régionalisation des compétences législatives en matière de bien-être animal est un second moteur des évolutions en cours. À l'exception des questions de santé animale ${ }^{9}$ demeurant fédérales, la matière du bien-être animal est désormais transférée à la compétence de Régions aux contextes socio-culturels et aux majorités politiques très différents. À partir du $1^{\text {er }}$ juillet $2014^{10}$, ce sont les Régions flamande, wallonne, bruxelloise qui acquièrent compétence pour modifier la loi fondatrice, anciennement fédérale, du 4 août 1986, jusqu'à la réformer globalement.

La loi du 14 août 1986 n'est pas abrogée pour autant, mais comporte temporairement trois versions distinctes, au gré de décrets régionaux nouveaux. La Région wallonne a ainsi recodifié cette matière dans le Code wallon du

7. De 1995 à 2014, plusieurs propositions de loi furent déposées par des députés d'extrêmedroite ou populistes en vue d'interdire la pratique des abattages rituels. Le Conseil d'État, en sa section législation, y opposera d'emblée un avis négatif, en assemblée générale. V. X. Delgrange et H. Lerouxel, «L'accommodement belge éclot entre égalité formelle, légalité et neutralité », Revue du droit des religions, n 7, mai 2019, p. 109-129, sp. p. 121.

8. Règlement (CE) $n^{\circ} 1099 / 2009$ du Conseil du 24 septembre 2009 sur la protection des animaux au moment de leur mise à mort.

9. Le flou des frontières entre les catégories «santé» et «bien-être animal» suscite diverses difficultés nouvelles dans la répartition des compétences entre les différents législateurs.

10. Date d'entrée en vigueur de la loi spéciale du 6 janvier 2014 relative à la sixième réforme de l'État: Moniteur belge [ci-après M.B.], 31 janv. 2014. 
bien-être animal du 4 octobre 2018, qui n'abrogeait toutefois pas immédiatement la loi de 1986 pour la Wallonie.

Les dossiers législatifs vont se multiplier et se disperser. Ainsi, l'interdiction de l'élevage des animaux à fourrure ${ }^{11}$, la stérilisation obligatoire des chats $^{12}$, puis l'interdiction des animaux sauvages de cirque et des animaux de foire ${ }^{13}$, l'interdiction de couper les queues des chiens ou des chevaux ${ }^{14}$, l'interdiction des spectacles de rapaces ou encore l'interdiction d'avoir des relations sexuelles avec des animaux ${ }^{15}$, etc., vont se voir compléter par des législations nouvelles, concernant les garanties pour les animaux de liberté de mouvement, d'alimentation, d'hébergement, de transport, d'absence de souffrance... Le commerce et l'élevage sont de plus en plus encadrés. Les publicités pour donation ou commercialisation sont limitées à des revues spécialisées, sous des conditions strictes ${ }^{16}$. Des dizaines d'interdictions nouvelles sont édictées en toute matière, selon les Régions. On notera encore un renforcement des contraintes et limitations en matière d'expérimentation animale, à travers un vaste ensemble de règles nouvelles, dont le Code indique qu'elles couvrent désormais également «les formes foetales de mammifères à partir du dernier tiers de leur développement normal ». Enfin, plus généralement, une innovation du législateur wallon tient à la création d'un «permis de détention d'animaux», que chaque résident détient de plein droit et de manière immatérielle, mais qui peut être retiré en cas d'infractions spécifiques.

11. Décret wallon du 21 janvier 2015 modifiant la loi du 14 août 1986 relative à la protection et au bien-être des animaux afin d'interdire la détention d'animaux à des fins exclusives ou principales de production de fourrure: M.B., 30 janv. 2015.

12. Arrêté du Gouvernement wallon du 15 décembre 2016 relatif à la stérilisation des chats domestiques: M.B., 2 janv. 2017.

13. Seuls les animaux faisant partie de la liste fixée à l'annexe 2 de l'arrêté du Gouvernement wallon du 24 juillet 2018 relatif à l'agrément des parcs zoologiques et fixant la composition et le fonctionnement de la Commission wallonne des parcs zoologiques peuvent aujourd'hui figurer dans les cirques de Wallonie ou encore les expositions itinérantes. Les animaux exotiques y sont désormais interdits. Un nouvel article 6ter introduit dans la loi de 1986 a interdit les poneys et chevaux de foire au $1^{\text {er }}$ janvier 2019.

14. Arrêté royal du 17 mai 2001 relatif aux interventions autorisées sur les vertébrés pour l'exploitation utilitaire de l'animal ou pour limiter la reproduction de l'espèce: M.B., 4 juill. 2001, modifié par l'arrêté royal du 17 décembre 2008: M.B., 19 janv. 2009.

15. Art. $359^{\circ}$ de la loi du 4 août 1986, introduit après que la cour d'appel d'Anvers a estimé qu'avoir de telles relations sexuelles n'impliquait ni le dol spécial requis ni une omission fautive visée par la loi en l'état (Anvers, 8 nov. 2006: Juristenkrant, n 40, 2006, p. 12, cité par F. Dossche (éd.), op. cit., p. 242).

16. Le décret a toutefois été partiellement annulé sur ce point par arrêt de la Cour constitutionnelle $n^{\circ}$ 10/2021 du 21 janvier 2021: certaines de ces restrictions de publicité étant estimées incompatibles avec les garanties de liberté d'expression. 
Concernant la mise à mort, un étourdissement préalable est requis de façon générale, sauf les cas $« 1^{\circ}$ de force majeure; $2^{\circ}$ de pratiques de la chasse ou de la pêche; $3^{\circ}$ de lutte contre les organismes nuisibles; $4^{\circ}$ d'actions de mise à mort prévues en vertu de la loi sur la conservation de la nature». Le Code wallon confirme ainsi implicitement, par cette disposition, l'abrogation, adoptée l'année précédente par un décret du 18 mai 2017, de l'exemption historique d'étourdissement en cas d'abattage rituel ${ }^{17}$.

Il en va de même en Région flamande: à défaut d'avoir substitué un code flamand du bien-être animal à la version flamande de la loi du 4 août 1986, la Flandre a elle aussi étendu à de nombreuses reprises les normes de protection animale. En matière d'abattage rituel, un décret du 7 juillet 2017 prévoit l'abrogation des exemptions d'étourdissement pour rite religieux, selon les mêmes formules que la Région wallonne.

En Région de Bruxelles, l'extension de la protection animale a également été soutenue par diverses ordonnances modifiant la loi du 4 août 1986. En revanche, les contextes démographiques, économiques et politiques de Bruxelles ont jusqu'à présent conduit au maintien d'une exemption au bénéfice des abattages rituels.

\section{VASTE LÉGISLATION MAIS RARE JURISPRUDENCE}

Avant d'examiner les termes des débats juridico-politiques régionaux concernant le statut juridique des formes d'abattage rituel, on voudrait indiquer que ces longues évolutions de la protection législative du bien-être animal en Belgique laissent proportionnellement peu de traces en jurisprudence. Florence Dossche, dans un ouvrage important déjà cité ${ }^{18}$, s'inquiète de cette apparente faiblesse jurisprudentielle et redoute d'y déceler la trace d'un

17. Décret du 18 mai 2017, modifiant les articles 3, 15 et 16 et insérant un article 45ter dans la loi du 14 août 1986 relative à la protection et au bien-être des animaux: M.B., $1^{\text {er }}$ juin 2017, dont l'entrée en vigueur, fixée initialement au $1^{\text {er }}$ juin 2018, a été reportée au $1^{\text {er }}$ septembre 2019. Une abrogation similaire de l'exemption pour abattage religieux a été adoptée en Flandre par le décret flamand du 7 juillet 2017: M.B., 18 juill. 2017, en vigueur le $1^{\text {er }}$ janvier 2019. Le décret flamand prévoit temporairement la possibilité d'un étourdissement post-stunning, qui initialement avait été discuté également au Parlement de Wallonie, mais n'a finalement pas été inséré dans le décret wallon. L'explication fournie était d'éviter une discrimination entre les éleveurs de différentes espèces et de prolonger un délai unique permettant d'assurer la réversibilité des étourdissements de façon uniforme. La Région bruxelloise n'a pas à ce jour abrogé l'exemption pour abattage religieux.

18. F. Dossche, «Répression de la maltraitance animale. États des lieux et perspectives», in F. Dossche (éd), op. cit., p. 231-283, qui cite également une communication du 
manque d'effectivité des législations. Si les cours et tribunaux ont été amenés à statuer sur des cas fort divers, dont le trait commun est souvent l'horreur des faits, on constate l'absence de contentieux à connotation religieuse, hors les recours constitutionnels récents liés au statut des abattages rituels.

Un seul cas publié datant de 1994 traite spécifiquement de religion. Il s'agissait d'un recours en annulation soumis au Conseil d'État. Le requérant affirmait que sa religion «lui enjoignait d'aimer les animaux comme les hommes» et que pour cette raison «il recueillait des animaux errants dans sa maison, mais comme il n'était pas assez riche pour les accueillir tous, il leur distribuait de la nourriture sur la voie publique et autres lieux»: pour le requérant, "nourrir les animaux sur la voie publique et ailleurs [était] [...] un acte de foi, une "obligation" religieuse et une manifestation extérieure $\mathrm{du}$ culte que le [règlement communal] [venait] interdire par une mesure préventive et définitive, d'ordre général et permanent $[\ldots] »$. Pour rejeter le recours en annulation du règlement communal, le Conseil d'État se borne à indiquer, laconiquement, que ce règlement constitue une mesure de salubrité et de sécurité publiques qui satisfait aux conditions imposées par l'article 9 de la Convention européenne et «peut dès lors constituer une restriction admissible de la liberté de manifester sa religion ou ses convictions ${ }^{19}$ ».

D'autres types de jurisprudence qui nous semblent mériter attention ici concernent des usages culturels locaux, comme la pratique de la capture d'oiseaux par tenderie ${ }^{20}$, ou encore celle de l'ingestion de poissons vivants dans du vin lors d'une fête dite du craquelin ${ }^{21} \ldots$ À son propos, la Cour de cassation, estimant «que la mort des poissons n'était pas "sans nécessité" eu égard à leur espérance de vie réduite et au contexte historico-culturel dans lequel les faits s'étaient produits», avait jugé en 2002 que la loi belge n'interdisait pas de tenir compte d'une perspective culturelle et historique pour justifier la mort d'un animal dans certains $\operatorname{cas}^{22}$.

21 mars 2018 cosignée par 33 associations protectrices des animaux qui regrettait le «laxisme de la justice face aux mauvais traitements sur animaux».

19. CE, 19 janv. 1994, Delbouille: L-L. Christians, «D'un conflit entre un impératif religieux ou de conscience et une norme étatique», Revue régionale de droit 1994, p. 312-324.

20. V. par exemple une poursuite devant le tribunal correctionnel de Verviers en 2019, évoquée dans la presse nationale (La Dernière Heure, 21 oct. 2019).

21. F. Dossche, «Répression de la maltraitance animale...», art. cit., p. 242.

22. Cass., 5 nov. 2002, R.G. no 9.02.0042.N : Pasicrisie 2002, p. 2095, cité par F. Dossche, art. cit., p. 242. 


\section{ACCÉLÉRATION SYMBOLIQUE D'UN BIEN-ÊTRE ANIMALIER SÉCULARISÉ : DIFFRACTION ET POLARISATION SUR LES DROITS HUMAINS DES MINORITÉS RELIGIEUSES}

Les législations belges ont longtemps maintenu hors de leur champ d'application les abattages soumis à des rites religieux, à l'origine essentiellement juifs, puis progressivement islamiques à partir des années 1970. La sécularisation de la société belge, la montée en puissance de l'intérêt pour le bien-être animal et une polarisation des questions liées à l'islam semblent avoir lentement convergé jusqu'à un point de bascule, en 2014. Les débats se focalisent et s'accélèrent. Sont proclamées inacceptables les exemptions historiques au bénéfice d'abattage sans étourdissement, et inaudibles tous débats sur de quelconques évolutions progressives des pratiques religieuses. Dans toute la Belgique? Non. En Flandre et en Wallonie. Une Région fait exception jusqu'ici, en raison de son contexte social, économique et politique: la Région de Bruxelles-Capitale.

Les pages qui suivent seront consacrées à la présentation des principaux arguments qui ont structuré juridiquement ce basculement, tout en notant qu'il ne s'agit pas ici d'entrer dans un débat expert sur le fond de la souffrance animale, ni non plus d'anticiper, en droit, l'issue des recours décisifs actuellement pendants devant la Cour constitutionnelle belge.

Trois éléments nous semblent particulièrement marquants et baliseront les arguments en présence. Tout d'abord, l'opposition permanente du Conseil d'État, à travers une succession d'avis, y compris en assemblée générale, qui mettent en garde constante contre la disproportionnalité envers la liberté de religion que constituerait une abrogation abrupte des exemptions religieuses. Ensuite, le refus des parlementaires de se soumettre à ces avis, en avançant plusieurs arguments plaidant pour une perception plus équilibrée de la norme nouvelle. Enfin, l'originalité de la mesure clé de ce nouvel équilibre allégué: la garantie octroyée aux seuls abattages religieux de la nature réversible de l'étourdissement préalable qui sera pratiqué. Perpétuant ainsi un régime spécifique pour les abattages religieux, certes plus limité, les nouvelles législations échapperont-elles pour autant à un reproche de disproportionnalité? Quelles sont les marges propres du droit constitutionnel belge, à la lumière des contextes locaux? On balise ici les divers arguments évoqués entre légistes et juristes. 


\section{LA HIÉRARCHIE CONSTITUTIONNELLE ENTRE DROITS DE L'HOMME ET INTÉRÊTS DE L'ANIMAL}

Depuis l'origine, le Conseil d'État, en sa section de législation, a constamment ${ }^{23}$ rappelé la priorité juridique des droits humains fondamentaux garantis par la Constitution et la Convention européenne des droits de l'homme, sur les intérêts, certes légitimes, à voir les animaux protégés contre toute souffrance. Le Conseil d'État a toujours estimé que «la suppression de la dérogation à l'exigence d'étourdissement préalable en cas d'abattage rituel, si elle poursuit l'objectif légitime de mieux assurer le bien-être des animaux, porte une atteinte disproportionnée à la liberté de religion consacrée par l'article 9 de la Convention».

Il reste toutefois que les garanties des droits de l'homme, en ce compris la liberté de religion (et en particulier de pratiques religieuses) sont susceptibles d'être limitées par des intérêts publics précisés notamment par l'alinéa 2 de l'article 9 de la Convention européenne des droits de l'homme. C'est alors l'argument principal de la proportionnalité des mesures restrictives qui devient le coeur de plusieurs discussions, de nature différente.

\section{LA POSSIBILITÉ D'IMPORTATION DE VIANDES RELIGIEUSEMENT CONFORMES}

Dans la ligne de l'arrêt Cha'are Shalom ve Tsedek de la Cour européenne des droits de l'homme, abondamment cité par les parlementaires ${ }^{24}$, la possibilité d'acquérir à l'étranger une alimentation religieusement conforme est un argument qui a été souligné par le débat parlementaire en faveur d'un respect strict de la liberté de religion. L'invocation explicite ${ }^{25}$ de l'article 26 paragraphe 4 du règlement européen s'inscrit dans la même ligne: l'accès à des viandes religieusement conformes serait automatiquement garanti dès lors que cette disposition prohibe toute interdiction à l'importation de viande abattue

23. Y compris en assemblée générale, le Conseil d'État, en sa section législation, a rendu au moins six avis sur ces questions (V. note 5).

24. CEDH, 27 juin 2000, $\mathrm{n}^{\circ}$ 27417/95, Cha'are Shalom Ve Tsedek c. France, § 80.

25. Dans son dernier avis, le Conseil d'État renvoie aux marges possibles du droit européen et indique ne pas apercevoir dans le projet initial, comment, «en conformité avec la marge de manœuvre que ménage l'article 26, paragraphe 2 , du règlement, les aménagements nécessaires ont été recherchés pour réaliser un juste équilibre entre les intérêts contradictoires en jeu». 
sans étourdissement. La Cour constitutionnelle ne souscrit pas d'emblée à l'effet mécanique de cet argument. Elle en souligne très pragmatiquement les aléas, d'un simple point de vue régional. Elle indique en effet, dans son premier arrêt sur le décret flamand du 7 juillet 2017 que l'interdiction wallonne des abattages rituels sans étourdissement, adoptée deux mois plus tôt par le décret wallon du 18 mai 2017, devrait être prise en compte, pour mesurer la capacité des populations d'accéder réellement à des viandes conformes ${ }^{26}$. Dans son avis déposé au Parlement de Wallonie, le constitutionnaliste Christian Behrendt partage les mêmes mises en garde, même si elles sont moquées par certains parlementaires ${ }^{27}$. Les débats ne relèvent d'ailleurs pas que la Cour européenne, dans son arrêt Cha'are Shalom ve Tsedek, prenait elle-même soin de noter le caractère aisé de l'importation à Paris de viandes conformes provenant d'une Belgique guère éloignée ${ }^{28}$. L'existence de marchés de proximité, belges ou étrangers, n'est pas discutée ${ }^{29}$. Les débats parlementaires ont en revanche évoqué une idée proche, quoique sans s'y attarder ni procéder à d'autres vérifications: à savoir que les prix ${ }^{30}$ à l'importation des viandes conformes devraient rester probablement inchangés...

\section{PROCÉDURE PARLEMENTAIRE ET DIALOGUE AVEC LES PARTIES CONCERNÉES ET LES EXPERTS}

À la différence des travaux du Parlement flamand qui avaient intégré d'emblée les auditions des cultes concernés, les débats parlementaires wallons ont

26. Cour constit., 4 avr. 2019, $\mathrm{n}^{\circ}$ 53/2019 et $\mathrm{n}^{\circ} 52 / 2019$, pt B.24.4.

27. P. W. - C.R.I. no 20 (2016-2017), 17 mai 2017, p. 18.

28. Sur l'importance de cette fluidité "géographique» pour évaluer l'accès à des intérêts fondamentaux, V. nos analyses: L.-L. Christians, «La liberté de conscience et de formes de vie dans la concurrence des systèmes nationaux. Globalisation et proximité seconde dans la jurisprudence européenne des droits de l'homme», in M. E. Ancel, L. D'Avout, J. C. Fernandez Rosas, M. Goré, J. M. Jude (éd.), Le droit à l'épreuve des siècles et des frontières. Mélanges en l'honneur du Professeur Bertrand Ancel, Paris, LGDJ, 2018, p. 413-430.

29. La situation des pays proches de la Belgique est donc particulièrement importante. Par ex., sont à prendre en compte de ce point de vue les débats qui animent les Pays-Bas. M. DE BLoIS, «Een verbod op de religieuze slacht is in strijd met dde godsdienstvrijheid », NTKR, Tijdschrift voor Recht en Religie, $n^{\circ}$ 2020/4, p. 131-146.

30. Une des co-auteurs de la proposition de réforme indique simplement que «nous considérons que les prix de produits halal ou kasher, le cas échéant, n'augmenteront pas et qu'en conséquence aucun fidèle ne sera privé de la possibilité de se procurer et de manger une viande jugée conforme aux prescriptions religieuses [...] On concilie les principes à la fois économiques de grand marché, les principes religieux, et je pense que la Wallonie peut avoir valeur d'exemple, peut être pionnière, cela peut être aussi un label de qualité pour nos abattoirs [...]»: P. W. - C.R.I.C. n 132 (2016-2017), 20 mars 2017. 
été extrêmement vifs lorsqu'il s'est agi de procéder in extremis à de telles auditions, formellement absentes du processus parlementaire officiel. Certes, les parlementaires avaient indiqué avoir rencontré informellement toutes les parties prenantes et ne voyaient dès lors plus la nécessité de les entendre solennellement. Mais là encore, les positions du Conseil d'État étaient différentes, insistant précisément sur l'importance de ce type de dialogue avec toutes les parties prenantes en vue d'une évolution progressive et accompagnée ${ }^{31}$. Dans un premier temps, seules des contributions écrites ont été sollicitées des cultes, tandis que des experts du bien-être animal et de la Fédération belge de la viande allaient être entendus. Toutefois, note la politologue Caroline Sägesser, devant les protestations du président du Consistoire central israélite de Belgique, la commission décida d'auditionner également un représentant de cet organe, ainsi qu'un membre de l'Exécutif des musulmans de Belgique. L'audition du président du consistoire juif s'est révélée extrêmement tendue après qu'il eut évoqué l'histoire juive durant la dernière guerre mondiale et qu'il eut indiqué que les nazis avaient été les derniers à avoir interdit l'abattage rituel en Belgique ${ }^{32}$. La sérénité des parlementaires fut mise à grande épreuve au gré d'escalades verbales diverses et de l'afflux de courriers polémiques. Les études scientifiques et rapports experts ont fait l'objet des mêmes controverses ${ }^{33}$. Les représentants du culte islamique, et les documents déposés par eux, furent moins polarisés ${ }^{34}$. Et c'est précisément lors des auditions avec ceux-ci qu'a pu émerger un débat sur la variété des formes d'étourdissement (irréversible, réversible, ou post-cutting). La variété des usages islamiques à travers le monde fut précisée et attestée. Il y apparut, aux yeux des parlementaires, que l'étourdissement réversible pouvait être raisonnablement considéré comme compatible avec certaines interprétations religieuses et usages islamiques ${ }^{35}$. D'emblée le

31. «Le meilleur moyen d'élaborer de telles mesures est le dialogue, qui nécessite une ouverture aux alternatives de part et d'autre»: CE, avis, n' 59484/3, p. 16 [notre traduction].

32. Les comptes rendus parlementaires reproduisent les propos tenus: «Rappelons que la dernière fois où l'on a voulu porter atteinte, en Belgique, à l'abattage selon le rite, en ce qui concerne les juifs, c'était en octobre 1940, par l'occupant nazi.»: P. W. - C.R.I.C. $\mathrm{n}^{\mathrm{o}} 158$ (2016-2017), 20 avr. 2017, p. 7. Le ton était précédemment monté dans le débat public jusqu'à voir l'association Gaia évoquer, dans certains clips télévisés, certains rapprochements entre l'abattage religieux des moutons et l'holocauste commis par les nazis: https://www.lalibre.be/lifestyle/magazine/abattage-rituel-campagne-choc-de-gaia525cdfea357090649b79b4fd [consulté le 21 juin 2021].

33. V. M. Denis, Intervention au nom du Groupe socialiste: P. W. - C.R.I. no 20 (20162017), 17 mai 2017, p. 8.

34. Un autre débat a concerné la qualité de la saignée: P. W. - C.R.I. no 20 (2016-2017), 17 mai 2017 , p. 9.

35. Le député CDH Josy Arens, co-auteur de la proposition de loi, résumera a posteriori la procédure de la façon suivante: «La machine médiatique et la mobilisation citoyenne 
président du consistoire juif indiqua que ce type d'étourdissement demeurait totalement incompatible avec les prescrits du judaïsme ${ }^{36}$. Cette divergence religieuse concernant les dispositifs réversibles d'étourdissement méritait une attention des parlementaires. Avant d'examiner ce point, il convient toutefois de s'arrêter sur les arguments échangés à propos de la possibilité même de discuter d'interprétations de prescrits religieux au sein du débat parlementaire.

\section{L'ANALYSE DES PRESCRITS CONFESSIONNELS ET DES USAGES RITUELS}

Faisant valoir son étonnement face à certains arguments parlementaires évoquant l'absence de prescrits religieux interdisant explicitement l'étourdissement préalable, ou telle ou telle interprétation religieuse ${ }^{37}$, la section législation du Conseil d'État insiste tout particulièrement «sur le fait qu'il n'appartient en principe pas aux autorités publiques de se prononcer sur la légitimité des croyances religieuses ou sur les modalités d'expression de celles-ci, et qu'elles n'ont donc pas à porter d'appréciation sur la justesse théologique des convictions de fidèles ou de courants déterminés d'une religion $^{38} »$. Il reste qu'une certaine variété des usages religieux a été confirmée en islam par le représentant du culte, et que les parlementaires ont pu tout autant tenir cette considération comme un élément factuel contribuant à dessiner un espace de compromis crédible pour une partie des fidèles. On pourrait toutefois également interpréter cette ambivalence des discussions relatives aux «normes» religieuses comme un désintérêt envers le principe de l'autonomie personnelle des croyances et envers la nature avant tout

se sont quelque peu emballées et, pour préserver la nécessaire sérénité de nos débats, notre commission, en accord avec M. le Ministre Di Antoni (CDH), a fait le bon choix d'entendre officiellement - puisque officieusement, nous avions entendu tout le monde les différents acteurs concernés.»: P. W. - C.R.I. nº 20 (2016-2017), 17 mai 2017, p. 5.

36. Des débats doctrinaux au sein de la pensée juive se sont manifestés, indiquant certaines tensions externes au Consistoire de Bruxelles: V. par ex., H. ROSENBERG, «Als het moet, is verdoofd ritueel slachten ook koosjer en halal - Een pleidooi voor een meer genuanceerd joods standpunt» [S'il le faut, l'abattage rituel avec étourdissement est également kasher et halal - un plaidoyer pour une position juive plus nuancée], Recht, Religie en Samenleving, $n^{\circ}$ 2015/1-2, p. 61-107, ou encore N. ZomERSZTAJN, «L'obligation d'étourdissement est conforme au droit européen», 10 févr. 2021: https://www.cclj.be/actu/obligation-etourdissement-est-conforme-au-droit-europeen [consulté le 21 juin 2021].

37. Une parlementaire a ainsi déclaré en séance: «Concernant le prescrit religieux, la liberté religieuse, aucun dieu n'a demandé que les animaux souffrent inutilement et on peut aussi évoluer au $\mathrm{xxI}^{\mathrm{e}}$ siècle dans la réflexion pour aller à la rencontre d'un compromis. »: P. W. - C.R.I.C. no 132 (2016-2017), 20 mars 2017, p. 7.

38. CE, sect. légis., avis $\mathrm{n}^{\circ} 60870,2017$, p. 14. L'intérêt des parlementaires pour une discussion sur l'orthodoxie des pratiques religieuses demeure prégnant. 
individuelle du régime des droits fondamentaux, au profit d'une approche de l'abattage imputée au régime collectif des cultes (en l'occurrence reconnus), et soumise à ce titre à la seule autorité interne des chefs de culte consultés. Une ambiguité que le débat parlementaire n'a pas levée.

\section{LA GARANTIE, OCTROYÉE AUX SEULS ABATTAGES RELIGIEUX, DE N'ÊTRE SOUMIS QU'À UN DISPOSITIF RÉVERSIBLE D'ÉTOURDISSEMENT}

Pour les législateurs régionaux wallon et flamand, il ne s'est pas seulement agi d'abroger l'exonération ancienne, mais aussi d'édicter une nouvelle norme spécifique aux abattages religieux, formulée en ces termes: «Lorsque la mise à mort d'animaux fait l'objet de méthodes particulières d'abattage prescrites ${ }^{39}$ par des rites religieux, le procédé d'étourdissement doit être réversible et ne peut entraîner la mort de l'animal». Cette norme nouvelle est au cœur du compromis élaboré par les parlementaires régionaux en débat avec certains représentants religieux ${ }^{40}$.

Si dans un premier temps les propositions de réforme avaient prévu que l'étourdissement exigible à l'avenir serait en toute circonstance de nature réversible, il a été rappelé aux parlementaires que la technique actuellement utilisée pour l'étourdissement (notamment la perforation de la boîte crânienne par un pistolet à tige) équivaut elle-même à une mise à mort. La généralisation d'un dispositif d'étourdissement réversible (par électronarcose), admise par une partie de l'économie islamique, était techniquement impossible en l'état des abattoirs belges, et notamment concernant les bovins. Deux modifications ont alors été apportées: la première fut de retarder l'entrée en vigueur des nouvelles normes pour assurer un délai d'adaptation non seulement aux communautés religieuses concernées, mais aussi aux abattoirs eux-mêmes en vue de réellement pourvoir garantir un dispositif d'étourdissement réversible aux abattages religieux. On notera qu'en Flandre, pour les mêmes raisons de délai d'adaptation, a été admise à titre temporaire une

39. Un débat parlementaire a porté sur ce renvoi par le décret à des «prescrits» par des rites religieux. Un amendement a suggéré d'y substituer la formule «dans le cadre de rites religieux», pour éviter que le décret donne force à des "prescrits»: P. W. - C.R.I.C. $\mathrm{n}^{\circ} 172$ (2016-2017), 5 mai 2017, p. 14.

40. «Une alternative proportionnée» selon le développement de la proposition de loi initiale: Doc. parl., Ch., 2016-2017, n 781/1, p. 5, et de préciser: "Cette exigence de réversibilité ne sous-entend pas qu'un étourdissement non réversible pose des difficultés en termes de bien-être animal. L'objectif est bien de rassurer les communautés religieuses concernées.» (p. 6). 
forme d'étourdissement a posteriori (post-cut stunning), qui semble également acceptable pour certains courants religieux, juifs et musulmans. Cette piste n'a pas été adoptée en Wallonie.

Dès lors que la figure de l'abattage prescrit par des rites religieux demeure donc encore singulière en droit (en bénéficiant seule de la garantie de la nature réversible de l'étourdissement), les débats ont insisté sur la nécessité pour les autorités publiques de vérifier la nature religieuse de la demande, qui seule ouvre ce droit à garantie. Les risques de contentieux relatifs à cette vérification, déjà liés à l'ancienne exemption, se reconduisent ici, dans le cadre d'une garantie plus étroite.

Ce dispositif qui est présenté comme un compromis nouveau, issu d'un dialogue, reste toutefois traversé par plusieurs points d'incertitude: le premier, déjà souligné, tient à la disparité qu'il crée entre diverses interprétations croyantes, au sein de chaque tradition concernée, selon qu'elles sont ou non partagées entre les personnes individuellement impliquées d'une part et d'autre part leurs chefs de culte, seuls pressentis sur ce point dans les débats parlementaires. Une seconde limite est toutefois plus nette encore, dès lors que le dispositif alternatif retenu ne semble pertinent qu'aux yeux des communautés musulmanes, et en aucun cas pour le judaïsme représenté par ses autorités reconnues. Ceci était d'autant plus frappant que les différences qui ont été présentées entre les usages (et aussi les besoins) de ces deux communautés religieuses étaient loin d'être négligeables, ne fût-ce qu'au regard du nombre d'animaux réellement concernés. Les risques de "polarisation», que le Centre interfédéral pour l'égalité des chances et la lutte contre les discriminations avait par exemple évoqués dans un avis émis en $2018^{41}$, ne se jouent dès lors pas seulement entre certaines minorités religieuses et la majorité de la population, mais également dans la disparité du compromis garanti envers l'islam d'une part et absent envers le judaïsme d'autre part.

\section{INCERTITUDES ÉCONOMIQUES ET IMPACT JURIDIQUE}

Le débat parlementaire s'est fait plus discret quant à l'ampleur des arguments économiques, mais ceux-ci ont néanmoins transparu à plusieurs reprises. On les examine ici comme éléments potentiels d'un examen de proportionnalité.

41. UNIA, Avis sur l'interdiction générale de l'abattage sans étourdissement, 22 févr. 2018: https:// www.unia.be/files/Documenten/Aanbevelingen-advies/Interdiction_générale_de_l'abattage_ sans_étourdissement.pdf [consulté le 21 juin 2021]. 
Les difficultés techniques auxquelles sont confrontés les abattoirs ont été soulignées à plusieurs reprises: d'une part, quant à la capacité inéluctablement insuffisante d'abattage lors de certaines fêtes religieuses (à défaut de pouvoir encore recourir à des établissements temporaires ${ }^{42}$ ), mais d'autre part, bien plus largement, en raison des contraintes techniques qui ralentissent la modernisation technologique de ces abattoirs et n'ont pas permis aux parlementaires d'être certains que l'étourdissement réversible puisse être réellement généralisé à la hauteur des besoins en viandes religieusement conformes.

L'importance de la libre circulation des marchandises a été un point important du débat. On a déjà mentionné l'importance formelle prêtée à la possibilité pour les fidèles belges d'acquérir leur alimentation à l'étranger, mais d'autres considérations économiques n'ont pas manqué. La solution d'importation a ainsi été contestée, non pas seulement pour son caractère aléatoire au regard de la garantie du droit fondamental de liberté religieuse, mais aussi pour des raisons économiques internes. Ainsi, certes de façon minoritaire, des filières agricoles belges ont indiqué au Parlement combien elles redoutaient de voir leur échapper des marchés importants ${ }^{43}$.

Le risque de voir exporter une part non négligeable de telles viandes religieusement conformes, aussi bien avant qu'après la réforme législative, a également été pointé au cours du débat parlementaire ${ }^{44}$. Engorger les dispositifs belges pour ensuite procéder à des exportations ne peut-il être considéré comme un risque de limitation à la garantie interne de liberté de religion? Comment s'en prémunir? L'appui des pouvoirs publics à de telles filières d'exportation vers des marchés islamiques, rappelé par certains parlementaires, vient en quelque sorte renforcer une telle crainte.

42. En suite à l'arrêt CJUE, Gde ch., 29 mai 2018, C-426/16, Liga van Moskeeën en Islamitische Organisaties Provincie Antwerpen e.a.

43. Ainsi, l'avis du Conseil wallon du bien-être des animaux, du 31 mai 2016, opposé à l'abattage sans étourdissement, mentionne toutefois l'existence d'un avis minoritaire de la Fédération unie de groupements d'éleveurs et d'agriculteurs (FUGEA), selon lequel l'abattage sans étourdissement devrait continuer à pouvoir être pratiqué en Wallonie dans des abattoirs agréés, étant donné que l'arrêt de l'abattage sans étourdissement en Wallonie engendrerait des importations massives de viandes issues d'animaux abattus sans étourdissement et développerait l'abattage clandestin: CONSEIL WALLON DU BIEN-ÊTRE DES ANIMAUX, Avis relatif aux abattages sans étourdissement, 31 mai 2016, précit.

44. Une des co-auteurs de la réforme souligne que le problème des abattages sans étourdissement dépasse celui de la production de viande halal ou casher destinée aux consommateurs musulmans et juifs en Belgique, «en raison, depuis ces dernières années, du développement du marché halal à l'export et d'incitations publiques de soutien à ce marché, notamment l'Agence wallonne à l'exportation [et aux investissements étrangers (AWEX)]»: P. W. - C.R.I.C. no 77 (2016-2017), 16 janv. 2017, p. 10. 
La proportionnalité des nouvelles dispositions a également été discutée, à certains moments des débats, par une mise en regard dans une perspective plus globale, non pas géographique (comme dans l'import/export), mais diachronique (au long de la vie de l'animal). Si l'on se concentre sur les filières belges, quel sens y aurait-il à se focaliser sur les derniers instants de la vie de l'animal sans se préoccuper de son bien-être tout au long de sa vie? Ne convenait-il pas de décloisonner les grilles de proportionnalité et de comparer le bien-être animal tout au long de son parcours dans des filières religieuses ou locales d'élevage d'une part et des filières industrielles d'autre part? Ces remarques formulées par les écologistes ${ }^{45}$ d'une part et l'extrême-gauche ${ }^{46}$ d'autre part ont été renvoyées par le ministre à des projets ultérieurs. Seule l'extrême-gauche s'est finalement abstenue de voter la réforme. Au lendemain du vote, l'un des auteurs de la proposition de réforme a, quant à lui, publié une opinion dans la presse nationale indiquant sa sincère volonté de poursuivre l'examen de l'ensemble de la filière industrielle ${ }^{47}$.

\section{DIFFRACTIONS RÉGIONALES ET RISQUES DE RECOURS CONSTITUTIONNELS ET EUROPÉENS}

Dès lors que les législateurs wallon et flamand ont pu se libérer, grâce à la nouvelle répartition des compétences, des réticences de la Région bruxelloise à abroger l'exception d'abattage rituel, les débats avaient évolué rapidement. Les parlementaires wallons et flamands se sont cependant constamment confrontés au risque de recours constitutionnels et européens ${ }^{48}$. À plusieurs

45. V. amendements de Mme Ryckmans, Doc. parl., Ch., 2016-2017, $\mathrm{n}^{\circ}$ 781/3. Il convenait également que le Gouvernement soutienne au préalable la recherche scientifique en la matière. Ces suggestions n'ont pas été suivies lors des débats.

46. Le Parti du travail de Belgique (PTB) aurait préféré que soit mise en cause l'industrie alimentaire: "Se pencher sur les améliorations à apporter pendant tout le parcours de vie de l'animal, voilà la question prioritaire, et non résolue, loin de là.» Il estime scientifiquement contesté que l'abattage avec étourdissement préalable soit moins douloureux. Il soutient par ailleurs l'Exécutif des musulmans de Belgique qui estime «qu'il est possible de trouver un terrain d'entente si le Parlement wallon accepte d'attendre l'évolution des techniques avant de légiférer»: P. W. - C.R.I. no 20 (2016-2017), 17 mai 2017, p. 15.

47. J. ARENS, «Réformer nos abattoirs pour ne plus en avoir honte», LeSoir.be, 17 mai 2017. Il soulignait notamment que «l'esprit du rite religieux est plus défendable que le point de vue industriel qui transforme l'animal en objet $»$.

48. Ainsi, par ex., pour le Parti socialiste, le soutien tardif à la proposition est entouré de précautions, de conditions et de présages: «Si un État de droit comme le nôtre garantit constitutionnellement la liberté des pratiques de religion, il donne également les moyens légaux de contester telle ou telle norme. Il y a fort à parier que ce texte wallon devra subir 
reprises, les ministres en charge et certains députés ont indiqué leur crainte quant à l'insuffisance des solutions de compromis et des risques pris à trop se presser. Certains auteurs ont notamment souligné l'effet contre-productif de l'un des arguments récurrents des auteurs de la proposition de réforme, à savoir que des sondages auraient montré que neuf Belges sur dix sont opposés à l'abattage sans étourdissement. Le constitutionnaliste Matthias El Berhoumi n'hésita pas ainsi à conclure son commentaire législatif en soulignant que « ces arguments s'opposent frontalement à la logique des droits de l'homme qui ont précisément vocation à contenir les excès de la majorité. Ce faisant, les développements des propositions ne cherchent aucunement à démontrer la proportionnalité de l'ingérence dans la liberté religieuse ${ }^{49}$ ».

La Cour constitutionnelle belge a fini par être effectivement saisie. Par un premier arrêt $\mathrm{n}^{0} 53 \mathrm{du} 4$ avril $2019^{50}$, elle a adressé une question préjudicielle à la Cour de justice de l'Union, qui a estimé le 17 décembre $2020^{51}$ que l'exemption européenne dont bénéficie les abattages selon des prescrits religieux constitue une simple faculté maintenue au bénéfice des droits nationaux. Pour soutenir qu'il s'agit là d'une simple faculté, la Cour de Luxembourg fournit sa propre interprétation de la garantie européenne de liberté de religion, par référence à ce qu'elle estime être la jurisprudence de la Cour européenne des droits de l'homme et en se référant notamment à

un examen approfondi de la part de la Cour constitutionnelle. Celle-ci dira si nous avons atteint le point d'équilibre ou si notre texte limite de façon disproportionnée la pratique d'un culte. [...] Ce soir, je ne parlerai ni de victoire, ni de défaite.»: P. W. - C.R.I. no 20 (2016-2017), 17 mai 2017, p. 12.

49. M. El Berhoumi, «Abattage rituel: la liberté religieuse sacrifiée sur l'autel du bien-être animal ? », Justice en ligne, 13 févr. 2017: https://www.justice-en-ligne.be/Abattage-rituella-liberte [consulté le 21 juin 2021].

50. Cour constit., 4 avr. 2019, $\mathrm{n}^{\circ}$ 54/2019, recours en annulation du décret de la Région flamande du 7 juillet 2017 portant modification de la loi du 14 août 1986 relative à la protection et au bien-être des animaux, en ce qui concerne les méthodes autorisées pour l'abattage des animaux, introduits par le Consistoire central israélite de Belgique et autres, par l'ASBL «Unie Moskeeën Antwerpen» et l'ASBL «Islamitisch Offerfeest Antwerpen", par Marcel Lehrer et Nochem Jakobovics, par l'Exécutif des musulmans de Belgique et autres et par l'ASBL «Comité de Coordination des Organisations Juives de Belgique. Section belge du Congrès juif mondial et Congrès juif européen » et autres. Dans le même sens, Cour constit., 18 juill. 2019, n 115/2019, demandes de suspension partielle du décret de la Région wallonne du 4 octobre 2018 relatif au Code wallon du bien-être des animaux, introduites par Rabah Bouazza et autres et par Albert Guigui et autres. V. E. VERNIERS, «Dierenwelzijn in de rechtspraak van het Gronwettelijk Hof », Rechtskundig Weekblad, no 18, 2020-2021, p. 683-697.

51. CJUE, 17 déc. 2020, n C 336/19, sur renvoi préjudiciel de la Cour constitutionnelle de Belgique, 4 avr. 2019, précit.; V. S. WATtien, «Arrêt "Centraal Israëlitisch Consistorie van België" : l'interdiction de l'abattage rituel sans étourdissement préalable au regard du droit de l'Union européenne», JDE 2021, p. 228-231. 
l'argument de libre importation présent à la fois dans le règlement européen (CE) no 1099/2009 et dans l'arrêt strasbourgeois Cha'are Shalom ve Tsedek c. France.

Cette position européenne, dont le Conseil d'État indiquait à l'avance la précarité au regard du contexte belge, y compris quant aux risques d'insuffisance des importations, laisse la Cour constitutionnelle libre de déterminer les équilibres propres à ses garanties fondamentales nationales y compris au regard de ses contextes régionaux wallon et flamand.

Dans l'attente, la Région de Bruxelles-Capitale a quant à elle maintenu l'exemption en faveur des abattages religieux. À l'annonce de l'arrêt de la Cour de justice de l'Union et à l'approche de l'arrêt de la Cour constitutionnelle, le ministre bruxellois en charge a indiqué être prêt à rouvrir le débat ${ }^{52}$. Il reste que l'abstention de la Région de Bruxelles demeure liée non seulement à l'importante part musulmane de sa population et de son électorat, mais aussi précisément à l'avenir économique de ses activités d'abattage, vers lesquelles convergent désormais des flux d'autant plus importants qu'ils ne peuvent plus, à l'heure actuelle, se maintenir dans les deux autres régions.

\section{L'AMÉLIORATION DE LA FORMATION DES INTERVENANTS RELIGIEUX}

La Région de Bruxelles-Capitale, certes réticente à abroger l'exemption au bénéfice des abattages rituels n'a cependant pas délaissé d'autres voies d'amélioration. Ainsi, en 2015, un projet d'arrêté de gouvernement a été soumis à la section de législation du Conseil d'État. Il visait à exiger, à Bruxelles, que les «sacrificateurs religieux», même occasionnels, soient titulaires d'un certificat de compétence qui reprenne «les règles relatives au bien-être animal, celles-ci devant être connues et respectées par les sacrificateurs». Ce certificat serait délivré aux personnes ayant suivi une formation et présenté un test final auprès d'un/des organisme(s) désigné(s) par le ministre ou le secrétaire d'État compétent pour le bien-être animal. Le certificat devrait être obtenu avant que l'habilitation soit sollicitée auprès des représentants du culte concerné. Les sacrificateurs disposant d'au moins trois ans d'expérience dans un abattoir agréé seraient dispensés de la formation obligatoire.

52. Le ministre bruxellois du Bien-Être animal, Bernard Clerfayt, a ainsi invité en février 2021 les cultes pratiquant l'abattage rituel sans étourdissement «à discuter de ce sujet dans les semaines à venir»: https://www.rtbf.be/info/regions/bruxelles/ detail_bruxelles-abattage-sans-etourdissement-le-ministre-du-bien-etre-animal-invite-lescultes-concernes?id=10688510 [consulté le 21 juin 2021]. 
Le certificat vaudrait pour une durée de trois ans et serait renouvelable. Mais aux yeux du Conseil d'État, ni la loi du 4 août 1986, encore en vigueur, ni le règlement européen $n^{0}$ 1099/2009 n'habilitait aucunement le Gouvernement à soumettre les sacrificateurs religieux, même occasionnels, à de telles conditions complémentaires de formation ${ }^{53}$.

Le président du consistoire israélite, lors de son audition au Parlement wallon, est revenu en vain sur une telle proposition. Il insistait sur le fait que l'acceptation, par l'institution qu'il représentait, de soumettre ses intervenants religieux à un complément de formation, y compris au regard de nouvelles techniques scientifiques, constituait une offre majeure de compromis. Sa formule mérite d'être citée:

«Contrairement à ce que prétendait, avant-hier, dans La Libre Belgique, Mme la Députée Défraie, également présidente du Sénat, le consistoire a fait un grand pas, une concession importante. Je m'explique. Les abatteurs selon le rite sont désignés, jusqu'à présent, exclusivement et uniquement, par le consistoire. Le consistoire propose qu'à l'avenir, un organe régional public neutre confirme les compétences, en particulier techniques, des candidats abatteurs, avant de leur permettre d'exercer. Si le candidat est refusé, le consistoire devra proposer une autre personne. Cet examen de compétence devra être objectif ${ }^{54}$.»

Cette proposition ne fut pas tenue pour significative.

\section{CONCLUSIONS}

Dans le cadre d'une réforme globale du Code civil belge, de nouvelles dispositions de ce code prévoient depuis septembre 2021 que «les choses, naturelles ou artificielles, corporelles ou incorporelles, se distinguent des animaux. Les choses et les animaux se distinguent des personnes. Les animaux sont doués de sensibilité et ont des besoins biologiques. Les dispositions

53. CE, avis, 11 juin 2015, n 57522/3. Le Conseil d'État ne va pas jusqu'à invoquer comme argument l'autonomie des cultes concernés dans la désignation de leur personnel, mais se borne à invoquer l'absence de normes de rang législatif habilitant une norme administrative à instaurer une telle exigence. Qu'une compétence spécifique puisse être exigée, et qu'une formation doive être offerte par les États, au titre de l'article 21 du règlement européen, ne modifie en rien l'inadéquation d'un simple arrêté administratif belge tel que formulé.

54. Le président du consistoire commençait par remercier la commission d'avoir accepté de postposer l'audition en raison de la fête de la Pâque juive: P. W. - C.R.I.C. no 158 (20162017), 20 avr. 2017, p. 7. 
relatives aux choses corporelles s'appliquent aux animaux, dans le respect des dispositions légales et réglementaires qui les protègent et de l'ordre public ${ }^{55} »$. Il s'agit là d'une étape symbolique majeure. Il reste que depuis 2014, la matière du bien-être animal n'est plus de la compétence du législateur fédéral, mais bien de trois législateurs régionaux.

Or, ces législateurs régionaux sont confrontés à des contextes très différents, qu'ils soient politiques, économiques, démographiques ou socioculturels. Comment toutefois faire progresser la protection régionalisée du bien-être animal tout en prenant au sérieux les garanties constitutionnelles relatives à la liberté de religion, voire à l'autonomie des cultes, en particulier pour les croyances minoritaires?

Un vaste débat s'est relancé en Belgique faisant de l'abattage rituel un point apparemment prioritaire sur d'autres dossiers davantage liés à des usages majoritaires comme la chasse ou l'élevage industriel. Quels équilibres trouver? Comment les construire en dialogue avec les parties prenantes? Dans quels délais? Par des accompagnements mutuels ou par des interdictions formelles? Certaines Régions pouvaient-elles adopter des normes qui semblent avoir été conçues sur mesure pour l'islam et non pour le judaïsme? Suffisait-il de garantir par décret la réversibilité des étourdissements pratiqués dans le cadre de demandes religieuses, alors qu'aucune certitude n'est assurée quant à la disponibilité de tels moyens techniques dans les abattoirs? Suffisait-il d'évoquer l'interdiction européenne de tout obstacle formel à l'importation de viandes religieusement conforme sans évaluer la capacité de tels flux économiques, les distances géographiques impliquées ou encore les variations de prix qui peuvent en résulter? Le Conseil d'État a constamment estimé qu'une interdiction absolue de l'abattage sans étourdissement contreviendrait de manière disproportionnée à la liberté de religion des fidèles concernés.

Ce débat compliqué a été écarté durant près de cent ans par les parlementaires belges au titre de la primauté de la liberté de religion et du principe de sauvegarde des usages minoritaires juifs. Ce débat s'est réamorcé depuis 2015 devant les parlements régionaux wallon et flamand. Au gré de

55. Art. 3.38 et 3.39 du nouveau livre 3 du Code civil (loi du 4 février 2020: M. B., 17 mars 2020, en vigueur $1^{\text {er }}$ sept. 2021). V. notamment F. GLANSORFF, « Réflexions sur la codification du droit animalier» in F. Dossche (éd.), Le droit des animaux. Perspectives d'avenir, op. cit., p. 315-339; E. LANGENAKEN, «L'animal entre l'être et l'avoir, une schizophrénie humaine et juridique», ibid., p. 285-314 et «L'animal en droit civil: les amorces d'un nouveau statut», Journal des tribunaux 2016, p. 693-703. 
mutations fortes de la population belge, dont les sondages ont été évoqués aux parlements, les rapports entre droits humains et intérêts des animaux se recomposent. Mais pour quels équilibres? Ou quelles ruptures? Plus rapides envers les religieux qu'envers les chasseurs?

Les positions divergentes entre Régions belges mettent bien en lumière les aspects contextuels de ces questions. Et dès lors que la possibilité d'importation semble à certains une solution admissible (et garantie par le droit de l'Union), c'est une sorte de cascade contextuelle qui se trouve être prise en compte. Cette cascade n'est rien d'autre que la trace d'un phénomène croissant de globalisation qui porte aussi bien sur le droit que sur le religieux ${ }^{56}$. Cette globalisation ne peut se limiter à une réalité brute. Le principe de proportionnalité la soumet nécessairement à une approche qualitative et critique. Le dossier belge en laisse entrevoir deux interrogations prospectives: d'une part, d'un point de vue économique, comment mesurer le caractère raisonnable et équitable des flux d'importation entre les Régions, à partir des capacités limitées de la Région de Bruxelles, ou de capacités plus lointaines; d'autre part, du point de vue juridique, comment s'appliquera en Belgique la clause de standstill, par laquelle l'article 53 de la Convention européenne de sauvegarde garantit le respect des conditions constitutionnelles nationales plus élevées de protection des droits humains.

$\mathrm{Au}$ moment où se posent ces questions, un recours est pendant devant la Cour constitutionnelle de Belgique après une réponse préjudicielle de la Cour de justice de l'Union européenne qui laisse la Belgique seule devant ses choix.

56. V. par ex., J. A. Rovinsky, «Don't have a Cow, Flanders: Guidance for the European Court of Justice as it Considers the Flemish Parliament's Ban on Ritual Slaughter », University of Detroit Mercy Law Review, nº 97/1, 2019, p. 180-199; et nos analyses déjà citées, dans L.-L. Christians, «La liberté de conscience et de formes de vie dans la concurrence des systèmes nationaux...», art. cit. 